\title{
Dirac Matrices and the Dirac Matrix Description of Lorentz Transformations*
}

\author{
A. J. Macfarlane \\ Department of Physics, Syracuse University, Syracuse, New York \\ Received December 5, 1965
}

\begin{abstract}
This paper gives what is believed to be a new discussion of Dirac matrices and of the Dirac matrix description of Lorentz transformations. The five anticommuting quantities $\gamma^{J}(J=1,2,3,0,5)$ are treated on an equal footing and recognition of the rule for expressing the three-fold product $\gamma^{J} \gamma^{K} \gamma^{L}$ in terms of one- and two-fold products and of invariant "five-space" tensors $g^{J K}, \varepsilon^{J K L P Q}$ allows all kinds of multiplication and trace laws for Dirac matrices to be derived systematically. The "five-space" formalism for Dirac matrices affords a very convenient vehicle for the Dirac matrix description of de Sitter transformations of a space with quadratic form $g_{K L} x^{K} x^{L}$. By considering the subset of these which leave the coordinate $x^{5}$ invariant, the Dirac matrix description of Lorentz transformations is obtained. Not only does this description give the well-known formula for any Lorentz transformation matrix $L$ in terms of the matrix $S$, which enters the transformation law of a Dirac spinor $\psi(x)$ under $L$, it also gives an explicit and apparently new inverse formula expressing $S$ in terms of $L$.
\end{abstract}

\section{Introduction}

In this paper, we present what is believed to be a new discussion of Dirac matrices and of the Dirac matrix description of Lorentz transformations.

Our discussion of Dirac matrices differs from the standard one ${ }^{1}$ in that it treats on an equal footing the five anticommuting quantities $\gamma^{J} \equiv\left(\gamma^{\mu}, \gamma^{5}\right)$ and gives their defining relations in the form [5]

$$
\begin{gathered}
\gamma^{J} \gamma^{K}+\gamma^{K} \gamma^{J}=2 g^{J K} \\
\gamma^{J+}=\gamma^{0} \gamma^{J} \gamma^{0} \\
\gamma^{J} \gamma^{K} \gamma^{L}=g^{J K} \gamma^{L}-g^{J L} \gamma^{K}+g^{K L} \gamma^{J}-\frac{1}{2} \varepsilon^{J K L P Q} \gamma_{P} \gamma_{Q},
\end{gathered}
$$

* Research supported in part by the U.S. Atomic Energy Commision under Contract AT (30-1) 3399.

1 Most of the algebraic properties of Dirac matrices were obtained first by PAULI [1] and are reviewed in [2]. Alternatively one may refer to any of a large number of standard works on relativistic quantum mechanics and field theory, e.g. [3] and [4]. 
wherein all indices take on values $1,2,3,0,5$, the metric tensor has signature $(-,-,-,+,-)$, and $\varepsilon$ is the totally antisymmetric tensor. These defining relations clearly contain the same content as the usual description but the recognition of (1.3) yields a fast and systematic derivation of and compact forms for a wide variety of multiplication and trace laws. Translation of theselaws into space-time notation then provides a more complete and readily extensible list of the algebraic properties of Dirac matrices than seems to be elsewhere available (see [6]).

From the defining relations (1.1) and (1.3) it seems reasonable to suspect that the Dirac matrix fromalism yields a description of transformations in a five dimensional Euclidean space similar in nature to the quaternion or Pauli matrix description of rotations in three dimensional Euclidean space. This is indeed correct. One can thus obtain a Dirac matrix description of the group $R_{5}$ of real rotations in five dimensions. Just as the quaternion description of rotations rests on the homomorphism of the group $S U_{2}$ generated by the quaternions to the group $R_{3}$ of rotations in three dimensions, so also does the Dirac matrix formalism for $R_{5}$ rest on the well-known homomorphism (see [7], p. 141, as well as [8]) of $U S p_{4}$, the symplectic subgroup of the group $S U_{4}$ generated by the Dirac matrices, to $R_{5}$. It does not, however, suit the purposes of the present work to deal with the $R_{5}, U S p_{4}$ relationship but instead we deal with the de Sitter group $L_{4,1}$, which leaves invariant the form $g_{J K} x^{J} x^{L}$ of a pseudo-Euclidean space $E_{4,1}$ and the related symplectic subgroup $V S p_{4}$ of a group $S V_{4}$ of pseudo-unitary transformations generated by Dirac matrices. The prefix "pseudo" as used here in connection with $S V_{4}$ has the following sense. If $\zeta$ is a point of the complex vector space of four dimensions on which $S U_{4}$ and $S V_{4}$ act, $S U_{4}$ leaves invariant the positive definite form $\zeta^{+} \zeta$ so that $S \in S U_{4}$ satisfies $S^{+} S=1$. However, $S V_{4}$ leaves invariant the indefinite form $\zeta^{+} \gamma^{0} \zeta$, so that $S \in S V_{4}$ satisfies $S^{+} \gamma^{0} S=\gamma^{0}$, or $S^{ \pm} S=1$ where $S^{ \pm}=\gamma^{0} S^{+} \gamma^{0}$ is the adjoint of $S$ with respect to the indefinite metric (see e.g. [9] or [10]) $\gamma^{0}$; i.e. $S$ is pseudo-unitary or unitary with respect to the indefinite metric $\gamma^{0}$. In this paper, we present a detailed discussion of the homomorphism of $V S p_{4}$ to $L_{4,1}$, thereby providing a Dirac matrix description of $L_{4,1}$. The discussion follows the pattern of a previous paper [11] on the quaternion description of real and complex rotations in three dimensions and of Lorentz transformations. In particular, it gives not only an explicit formula for the mapping of $V S p_{4}$ on $L_{4,1}$, but also its more useful and less obvious inverse.

While the discussion of $L_{4,1}$ in terms of Dirac matrices is of intrinsic interest, the motivation for it is that one obtains a complete compact development of the Dirac matrix description of the group $L_{3,1}$ of Lorentz transformations from it simply by considering the subgroup $L_{3,1}$ of 
$L_{4,1}$ which leaves the coordinate $x^{5}$ invariant. The principal advantage of the present approach to the Dirac matrix formalism for $L_{4,1}$ over the conventional one, aside from the debatable remark that we are here probably looking at it within its most natural mathematical context, is as follows. Not only do we give the well-known formula expressing any Lorentz transformation in terms of the matrix $S(L)$, which enters the transformation law under $L$ of a Dirac spinor, $\psi(x) \vec{L} S(L) \psi(L x)$, but we also give explicitly the inverse formula for $S(L)$ in terms of $L$. This important result is believed to be new.

The reason for going to the Dirac matrix description of $L_{3,1}$ via that of $L_{4,1}$ is that the latter can be formulated in terms of the compact five dimensional space statement of the algebraic properties of Dirac matrices. Similarly our desire to get the Dirac matrix formalism for $L_{3,1}$ motivated our study of $L_{4,1}$ rather than $R_{5}$. Also the occurrence of $\zeta^{+} \gamma^{0} \zeta$ as the invariant form for $V S p_{4}$ is seen as the familiar $\bar{\zeta}$ of Dirac theory.

In section two, we discuss the algebra of Dirac matrices (multiplication, trace and completeness laws and $B$ and $D$ such that $\tilde{\gamma}^{J}=B \gamma^{J} B^{-1}$, $\left.\gamma^{J *}=D \gamma^{J} D^{-1}\right)$ in five space notation. In section three, the Dirac matrix description of $L_{4,1}$ is given, and, in section four, after a translation of the multiplication and trace laws of section two for Dirac matrices into space-time notation, our Dirac matrix description of Lorentz transformations is presented and briefly contrasted with the conventional discussion as given in connection with the Lorentz invariance of the Dirac equation.

\section{Dirac matrices}

Our discussion of Dirac matrices differs from the standard textbook discussion in that all five anticommuting Dirac matrices are treated on the same footing. Our Dirac matrices are in fact defined by

$$
\begin{gathered}
\gamma^{J} \gamma^{K}+\gamma^{K} \gamma^{J}=2 g^{J K} \\
\gamma^{J+}=\gamma^{0} \gamma^{J} \gamma^{0} \\
\gamma^{J} \gamma^{K} \gamma^{L}=g^{J K} \gamma^{L}-g^{J} \gamma^{K}+g^{K L} \gamma^{J} \\
-\frac{1}{2} \varepsilon^{J K L P Q} \gamma_{P} \gamma_{Q} .
\end{gathered}
$$

Herein all indices vary over the range $1,2,3,0,5$ and summation convention implies a sum over these values of a repeated index. The metric tensor $g^{J K}$ obeys

$$
g^{J K}=0, \quad J \neq K ; \quad g^{11}=g^{22}=g^{33}=-g^{00}=g^{55}=-1 ;
$$

and the totally antisymmetric tensor $\varepsilon$ is such that

$$
\varepsilon^{12305}=1 .
$$


Our description is equivalent to the usual one - for example (2.3) yields $\gamma^{0} \gamma^{5}=\gamma^{1} \gamma^{2} \gamma^{3}=$ or $\gamma^{5}=\gamma^{0} \gamma^{1} \gamma^{2} \gamma^{3}$; we have simply recognized the existence of the multiplication law (2.3). Products of four or more $\gamma$ 's can be expressed in terms of one and two fold products and the numerical tensors by iteration of (2.3) and use of familiar properties of $\varepsilon$ 's. Explicitly, for the four fold product we have

$$
\begin{aligned}
\gamma^{K} \gamma^{L} \gamma^{M} \gamma^{N}= & g^{K L} \gamma^{M} \gamma^{N}-g^{K M} \gamma^{L} \gamma^{N}+g^{K N} \gamma^{L} \gamma^{M}+ \\
& +g^{M N} \gamma^{K} \gamma^{L}-g^{L N} \gamma^{K} \gamma^{M}+g^{L M} \gamma^{K} \gamma^{N} \\
& -g^{K L} g^{M N}+g^{L N} g^{K M}-g^{L M} g^{K N}+\varepsilon^{K L M P} \gamma_{P} .
\end{aligned}
$$

In order to deal with a set of sixteen linearly independent Dirac matrices, we adjoin to 1 and $\gamma^{K}$, the ten independent quantities

which satisfy

$$
G^{L M}=\frac{1}{2} i\left[\gamma^{L}, \gamma^{M}\right]
$$

$$
\begin{aligned}
G^{L M} & =-G^{M L}, \\
G^{L M+} & =\gamma^{0} G^{L M} \gamma^{0} .
\end{aligned}
$$

We may easily now obtain from (2.3) and (2.4) multiplication rules involving the $G^{L M}$ :

$$
\begin{gathered}
\gamma^{K} G^{L M}=i\left(g^{K L} \gamma^{M}-g^{K M} \gamma^{L}\right)-\varepsilon^{K L M P Q} G_{P Q} \\
{\left[\gamma^{K}, G^{L M}\right]=2 i\left(g^{K L} \gamma^{M}-g^{K M} \gamma^{L}\right)} \\
G^{K L} G^{M N}=i\left(g^{L M} G^{K N}+g^{K N} G^{L M}-g^{K M} G^{L N}-g^{L N} G^{K M}-\right. \\
-g^{L M} g^{K N}+g^{K M} g^{L N}-\varepsilon^{K L M N P} \gamma_{P} \\
{\left[G^{K L}, G^{M N}\right]=2 i\left(g^{L M} G^{K N}+g^{K N} G^{L M}-g^{K M} G^{L N}-g^{L N} G^{K M}\right)}
\end{gathered}
$$

Also, we have the trace properties

$$
\begin{aligned}
\operatorname{Tr} \gamma^{K} & =0 \\
\operatorname{Tr} \gamma^{K} \gamma^{L} & =4 g^{K L} \\
\operatorname{Tr} \gamma^{K} \gamma^{L} \gamma^{M} & =0 \\
\operatorname{Tr} \gamma^{K} \gamma^{L} \gamma^{M} \gamma^{N} & =4\left(g^{K L} g^{M N}-g^{K M} g^{L N}+g^{K N} g^{L M}\right), \\
\operatorname{Tr} \gamma^{K} \gamma^{L} \gamma^{M} \gamma^{N} \gamma^{P} & =4 \varepsilon^{K L M N P} \\
\operatorname{Tr} G^{L M} & =0 \\
\operatorname{Tr} \gamma^{K} G^{L M} & =0 \\
\operatorname{Tr} G^{K} L G^{M N} & =4\left(g^{K M} g^{L N}-g^{K N} g^{L M}\right) \\
\operatorname{Tr} \gamma^{K} \gamma^{L} G^{M N} & =4 i\left(g^{K M} g^{L N}-g^{K N} g^{L M}\right) \\
\operatorname{Tr} \gamma^{K} G^{L M} G^{N P} & =-4 \varepsilon^{K L M N P}
\end{aligned}
$$


It is to be noted longer traces can be worked out easily without completely evaulating the corresponding products of $\gamma$ 's.

The completeness relation for the Dirac matrices in the present notation appears as

$$
\delta_{a b} \delta_{c d}+\left(\gamma_{K}\right)_{a b}\left(\gamma^{K}\right)_{c d}+\frac{1}{2}\left(G_{L M}\right)_{a b}\left(G^{L M}\right)_{c d}=4 \delta_{a d} \delta_{b c}
$$

Next we observe that the defining relations are form-invariant under the substitution ${ }^{2}$

$$
\gamma^{K} \rightarrow \tilde{\gamma}^{K}
$$

although not under $\gamma^{K} \rightarrow-\tilde{\gamma}^{K}$. As a consequence of (2.23) and the unitary nature of our $\gamma$ 's, it follows that there exists a unitary matrix $B$ such that

From (2.24), the result

$$
\tilde{\gamma}^{K}=B \gamma^{K} B^{-1}
$$

$$
\tilde{G}^{L M}=-B G^{L M} B^{-1}
$$

follows. As in the textbook discussion, we must have

$$
\widetilde{B}=-B
$$

as there can be only six linearly independent antisymmetric Dirac matrices. These six are $B$ and $B \gamma^{K}$, while all $B G^{L M}$ are symmetric. The antisymmetric matrix $B$ will be seen to play a crucial role in the ensuing discussion.

Finally from (2.24) and (2.2) we obtain

where $D$ is given by

$$
\gamma^{K *}=D \gamma^{K} D^{-1}
$$

$$
D=B \gamma^{0}
$$

\section{Dirac matrix description of de Sitter transformations}

The basis of the work of this section is equivalent to the wellknown homomorphism of the group $R_{5}$ of real rotations in a real Euclidean space $E_{5}$ of five dimensions to the group $U S p_{4}$ of unitary symplectic transformations in a unitary space $C_{4}$ of four dimensions. The group $R_{5}$ leaves invariant the positive definite form

$$
\left(x^{1}\right)^{2}+\left(x^{2}\right)^{2}+\left(x^{3}\right)^{2}+\left(x^{4}\right)^{2}+\left(x^{5}\right)^{2}
$$

in $E_{5}$. The group $U S p_{4}$ is the symplectic subgroup of $U_{4}$, the unitary group which leaves invariant the hermitian form $\zeta^{+} \zeta$ of $C_{4}$, i.e. $U S p_{4}$ alsoleavesinvariant the antisymmetric form $\tilde{\zeta} J \zeta$ where $J$ is antisymmetric. The matrix $B$ of (2.24) is the obvious choice of a suitable matrix $J$.

${ }^{2}$ In the following $\tilde{j}$ denotes the transposed matrix, $j^{*}$ the complex conjugate. 
Here, in view of our aim of ultimately obtaining the Dirac matrix description of Lorentz transformations, we consider not $R_{5}$ but the de Sitter group $L_{4,1}$ of real transformations in a pseudo Euclidean space $E_{4,1}$ five dimensions, i.e. the group of real transformations which leave invariant the indefinite quadratic form

$$
\begin{gathered}
-\left(x^{1}\right)^{2}-\left(x^{2}\right)^{2}-\left(x^{3}\right)^{2}+\left(x^{0}\right)^{2}-\left(x^{5}\right)^{2} \\
=g_{M N} x^{M} x^{N}
\end{gathered}
$$

We shall present, in terms of the formalism of the preceding section, a detailed analysis of the homomorphism of $L_{4,1}$ to a group $V S p_{4}$ of pseudo-unitary symplectic transformations in a pseudo-unitary space $C_{4}^{\prime}$. The group $V S p_{4}$ is the symplectic subgroup of the pseudo-unitary group $V_{4}$ which leaves invariant the indefinite form $\zeta^{+} \gamma^{0} \zeta$, i.e. $V S p_{4}$ consists of those four by four matrices $S$ which satisfy the pseudo-unitary condition $^{3}$

and the symplectic condition

$$
S^{+} \gamma^{0} S=\gamma^{0}
$$

$$
\widetilde{S} B S=B \text {. }
$$

It should be noted that as a result of (3.1) and (3.2), such $S$ also satisfy

with $D$ given by 2.28 .

$$
S^{*}=D S D^{-1}
$$

We shall confine our attention to the proper orthochronous de Sitter group, refer to it as $L_{4,1}$ and omit the qualifying adjectives hereafter. If $x^{M}$ is a point of $E_{4,1}$, then the elements $\mathscr{L}$ of $L_{4,1}$ are defined by

where

$$
x^{M} \rightarrow \hat{x}^{M}=\mathscr{L}^{M}{ }_{N} x^{N},
$$

$$
\begin{gathered}
\mathscr{L}^{M N}=\left(\mathscr{L}^{-1}\right)^{N M}, \\
\mathscr{L}^{00} \equiv 1, \quad \operatorname{det} \mathscr{L}=1,
\end{gathered}
$$

and $\mathscr{L}$ here stands for the matrix with $\mathscr{L}^{M}{ }_{N}$ at the intersection of the $M^{\text {th }}$ row and $N^{\text {th }}$ column. Clearly $\mathscr{L}$ leaves $g_{M N} x^{M} x^{N}$ invariant and involves ten independent real parameters.

To find the relation of $L_{4,1}$ to $V S p_{4}$, we begin by associating with each point $x^{K}$ of $E_{4,1}$ a four by four matrix $X$ defined by

$$
X=x_{K} \gamma^{K},
$$

3 The forms of (3.1) and (2.2) may be seen to be quite natural as follows. If we dealt with $U_{4}$, we should have been concerned with $C_{4}$, which has metric matrix 1 and should use hermitian $\gamma$ 's and unitary $S$. However, we deal with $V_{4}$ and $C_{4}^{\prime}$ which has indefinite metric $\gamma^{0}$, and use $\gamma^{\prime}$ 's obeying (2.2) and $S$ obeying (3.1). If we define $M \pm=\gamma^{0} M^{+} \gamma^{0}$ as the adjoint of a matrix $M$ with respect to the indefinite metric $\gamma^{0}$, then our $\gamma^{\prime}$ s obey $\gamma^{J \pm}=\gamma^{J}$ and $S$ obeys $S^{-1}=S \pm$, so that the transition $U_{4} \rightarrow V_{4}$ corresponds to the replacement of $M^{+}$by the adjoint $M \pm$ with respect to $\gamma^{0}$. 
the inverse relationship being

$$
x^{K}=\frac{1}{4} \operatorname{Tr}\left(\gamma^{K} X\right) .
$$

We then consider the effect of the transformation

$$
X \rightarrow S X S^{-1}
$$

where $S \in V S p_{4}$, i.e. obeys (3.1) and (3.2). We shall prove that (3.8) can be used to describe an element of $L_{\mathbf{4}, 1}$. Evidently it is firstly necessary that $S X S^{-1}$ be of the form $\hat{x}_{J} \gamma^{J}$ for some $\hat{x}_{J}$, for then we can define $\mathscr{L}$ by

$$
\hat{x}^{J}=\mathscr{L}^{J}{ }_{K} x^{K}
$$

and proceed to prove $\mathscr{L} \in L_{4,1}$. For $S X S^{-1}$ to be of the required form, the coefficients of 1 and $G^{L M}$ in its expansion with respect to a complete set of Dirac matrices must be zero, i.e. we must have

$$
\operatorname{Tr}\left(S X S^{-1}\right)=0
$$

which is trivially satisfied for any $S$, and

$$
\operatorname{Tr}\left(S X S^{-1} G^{L M}\right)=0 .
$$

That $S \in V S p_{4}$ indeed satisfies (3.10) emerges as a result of (3.2) as follows:

$$
\begin{aligned}
\operatorname{Tr}\left(S X S^{-1} G^{L M}\right) & =\operatorname{Tr}\left[\left(S X S^{-1} G^{L M}\right)^{\sim}\right] \\
& =\operatorname{Tr}\left[\tilde{G}^{L M} \tilde{S}^{-1} \tilde{X} \tilde{S}\right] \\
& =-x_{K} \operatorname{Tr}\left[B G^{L M} B^{-1} \tilde{S}^{-1} B \gamma^{K} B^{-1} \tilde{S}\right] \\
& =-x_{K} \operatorname{Tr}\left[G^{L M}\left(B^{-1} \tilde{S} B\right)^{-1} \gamma^{K}\left(B^{-1} \tilde{S} B\right)\right] \\
& =-\operatorname{Tr}\left(G^{L M} S X S^{-1}\right)=0 .
\end{aligned}
$$

Herein, (2.24) and (2.25) are used in the second line and (3.2) in the fourth. Conversely it can be proved that, if (3.10) is satisfied by pseudounitary $S^{\prime}$, then $S^{\prime}$ must obey a result of the form.

$$
\widetilde{S^{\prime}} B S^{\prime}=B e^{2 i \theta}
$$

and the undetermined phase can be eliminated by a redefinition $S^{\prime} \rightarrow S$ $=S^{\prime} e^{-i \theta}$, so that $S$ obeys (3.2).

Thus we consider the transformation

$$
X \rightarrow \hat{X}=S X S^{-1},
$$

where $S \in V S p_{4}$ and $\hat{X}=\mathscr{L}_{J}{ }^{K} x_{K} \gamma^{J}$. Since this holds for any point $x_{K}$ of $E_{4,1}$, we get

and hence

$$
\mathscr{L}_{J}^{K} \gamma^{J}=S \gamma^{K} S^{-1}
$$

$$
\mathscr{L}^{J K}=\frac{1}{4} \operatorname{Tr}\left(\gamma^{J} S \gamma^{K} S^{-1}\right) \equiv \mathscr{L}(S)^{J K}
$$


We must now prove that $\mathscr{L}$ as given by (3.12) satisfies (3.5) and hence belongs to $L_{4,1}$. Then, if the result

$$
\mathscr{L}\left(S_{1}\right) \mathscr{L}\left(S_{2}\right)=\mathscr{L}\left(S_{1} S_{2}\right)
$$

can be proved, it follows that (3.13) gives an explicit realization of the $2: 1$ homomorphism

$$
\pm S \leftrightarrow \mathscr{L}(S)
$$

of $V S p_{4}$ to $L_{4,1}$. It is convenient to establish (3.14) first. This is done directly as follows

$$
\begin{aligned}
\mathscr{L}\left(S_{1}\right)^{J}{ }_{K} \mathscr{L}\left(S_{2}\right)^{K}{ }_{M}= & \frac{1}{4}\left(S_{1}^{-1} \gamma^{J} S_{1}\right)_{b a}\left(\gamma_{K}\right)_{a b} \frac{1}{4}\left(\gamma^{K}\right)_{c d}\left(S_{2} \gamma_{M} S_{2}^{-1}\right)_{d c} \\
= & \frac{1}{16}\left(S_{1}^{-1} \gamma^{J} S_{1}\right)_{b a}\left(S_{2} \gamma_{M} S_{2}^{-1}\right)_{d c} \\
& {\left[\delta_{a b} \delta_{c d}+\left(\gamma_{K}\right)_{a b}\left(\gamma^{K}\right)_{c d}+\frac{1}{2}\left(G_{P Q}\right)_{a b}\left(G^{P Q}\right)_{c d}\right] } \\
= & \frac{1}{4} \operatorname{Tr}\left(S_{1}^{-1} \gamma^{J} S, S_{2} \gamma_{M} S_{2}^{-1}\right) \\
& =\mathscr{L}\left(S_{1} S_{2}\right)_{M}^{J} .
\end{aligned}
$$

To obtain the second line, the results $\operatorname{Tr}\left(S_{2} \gamma_{M} S_{2}^{-1}\right)=0$, and $\operatorname{Tr}\left(S_{2} \gamma_{M} S_{2}^{-1} G^{P Q}\right)=0$, which is equivalent to (3.10), have been used. Use of the completeness relation for Dirac matrices follows, leading to the desired result. We now turn to the other points in order. The reality of $\mathscr{L}$ follows use of $(\operatorname{Tr} M)^{*}=\operatorname{Tr} M^{+}$, and $\left[\mathscr{L}(S)^{-1}\right]^{J K}=\mathscr{L}(S)^{K J}$ follows use of $\mathscr{L}(S)^{-1}=\mathscr{L}\left(S^{-1}\right)$, which itself follows (3.14). Also $\mathscr{L}(S)^{00}=\frac{1}{4} \operatorname{Tr}\left(S S^{+}\right) \equiv 0$ implies that we are dealing with orthochronous $\mathscr{L}$. Finally to prove $\operatorname{det} \mathscr{L}=1$, we use the results

and

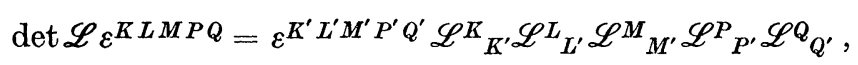

$$
\varepsilon^{K L M P Q} \gamma_{K} \gamma_{L} \gamma_{M} \gamma_{P} \gamma_{Q}=1
$$

which can be obtained from (2.4) and familiar properties of $\varepsilon$ 's. Details of the proof are

$$
\begin{aligned}
& \operatorname{det} \mathscr{L}=\operatorname{det} \mathscr{L} \varepsilon^{K L M P Q} \gamma_{K} \gamma_{L} \gamma_{M} \gamma_{P} \gamma_{Q} \\
& =\varepsilon^{K^{\prime} L^{\prime} M^{\prime} P^{\prime} Q^{\prime}}\left(\mathscr{L}^{K}{ }_{K^{\prime}} \gamma_{K}\right)\left(\mathscr{L}^{L}{ }_{L^{\prime}} \gamma_{L}\right)\left(\mathscr{L}^{M_{M^{\prime}}} \gamma_{M}\right)\left(\mathscr{L}^{P}{ }_{P^{\prime}} \gamma_{P}\right)\left(\mathscr{L}^{Q}{ }_{Q^{\prime}} \gamma_{Q}\right) \\
& =\varepsilon^{K^{\prime} L^{\prime} M^{\prime} P^{\prime} Q^{\prime}} S\left(\gamma_{K^{\prime}} \gamma_{L^{\prime}} \gamma_{M^{\prime}} \gamma_{P^{\prime}} \gamma_{Q^{\prime}}\right) S^{-1}=1 \text {, }
\end{aligned}
$$

use of (3.21) having been made.

To complete the discussion of $V S p_{4}$ and $L_{4,1}$, we need the formula inverse to (3.13). This shall be obtained by use of the consequence

$$
S 1 S^{-1} 1+S \gamma_{K} S^{-1} \gamma^{K}+\frac{1}{2} S G_{L M} S^{-1} G^{L M}=4 S\left(\operatorname{Tr} S^{-1}\right),
$$


of (2.24). First, we use (3.12) to evaluate the second term obtaining

$$
\begin{aligned}
S \gamma_{K} S^{-1} \gamma^{K} & =\mathscr{L}_{J K} \gamma^{J} \gamma^{K} \\
& =\operatorname{Tr} \mathscr{L}-i \Delta(\mathscr{L})
\end{aligned}
$$

where

$$
\Delta(\mathscr{L})=\mathscr{L}_{J K} G^{J K} .
$$

Next we evaluate the third term according to

$$
\begin{aligned}
S G_{L M} S^{-1} G^{L M}= & -S\left(\gamma_{L} \gamma_{M}-g_{L M}\right) S^{-1}\left(\gamma^{L} \gamma^{M}-g^{L M}\right) \\
= & -S \gamma_{L} \gamma_{M} S^{-1} \gamma^{L} \gamma^{M}+5 \\
= & -\mathscr{L}_{P L} \mathscr{L}_{Q M} \gamma^{P} \gamma^{Q} \gamma^{L} \gamma^{M}+5 \\
= & (\operatorname{Tr} \mathscr{L})^{2}-\operatorname{Tr} \mathscr{L}^{2}+2 i\left[\Delta\left(\mathscr{L}^{2}\right)-(\operatorname{Tr} \mathscr{L}) \Delta(\mathscr{L})\right]+ \\
& +\Phi(\mathscr{L}),
\end{aligned}
$$

where

$$
\Phi(\mathscr{L})=\varepsilon_{P Q L M R} \mathscr{L}^{P Q} \mathscr{L}^{L M} \gamma^{R} .
$$

In the last line of (3.19), we used (2.4) and (2.5). We may now combine (3.16), (3.17) and (3.19) to give

$$
\begin{gathered}
4 S\left(\operatorname{Tr} S^{-1}\right)=1+\operatorname{Tr} \mathscr{L}+\frac{1}{2}(\operatorname{Tr} \mathscr{L})^{2}-\frac{1}{2} \operatorname{Tr} \mathscr{L}^{2}+\frac{1}{2} \Phi(\mathscr{L}) \\
-i \Delta(\mathscr{L})+i \Delta\left(\mathscr{L}^{2}\right)-i(\operatorname{Tr} \mathscr{L}) \Delta(\mathscr{L}),
\end{gathered}
$$

and it remains to evaluate $\operatorname{Tr} S^{-1}$, which is easily done as follows. Since $S$ obeys (3.3), it must be of the form

$$
S=s+s_{K} \gamma^{K}+\frac{1}{2} i s_{P Q} G^{P Q},
$$

with $s, s_{K}, s_{P Q}=-s_{Q P}$ all real. Hence

$$
\operatorname{Tr} S=4 s=\operatorname{Tr} S^{*}=\operatorname{Tr} S^{+}=\operatorname{Tr} S^{-1},
$$

so that we get

$$
\begin{aligned}
\operatorname{Tr} S \operatorname{Tr} S^{-1} & =\left(\operatorname{Tr} S^{-1}\right)^{2} \\
& =1+\operatorname{Tr} \mathscr{L}+\frac{1}{2}(\operatorname{Tr} \mathscr{L})^{2}-\frac{1}{2} \operatorname{Tr} \mathscr{L}^{2}
\end{aligned}
$$

directly from (3.21). Hence finally we can write the required inverse formula in the form

$$
\begin{aligned}
S & =\left[F(\mathscr{L})+\frac{1}{2} \Phi(\mathscr{L})+i \Delta\left(\mathscr{L}^{2}\right)-i(1+\operatorname{Tr} \mathscr{L}) \Delta(\mathscr{L})\right][16 F(\mathscr{L})]^{-1 / 2} \\
& \equiv S(\mathscr{L}),
\end{aligned}
$$

where $\Phi$ and $\Delta$ are defined by $(3.20)$ and (3.11) and $F(\mathscr{L})$ is given by

$$
F(\mathscr{L})=[\operatorname{Tr} S(\mathscr{L})]^{2}=1+\operatorname{Tr} \mathscr{L}+\frac{1}{2}(\operatorname{Tr} \mathscr{L})^{2}-\frac{1}{2} \operatorname{Tr} \mathscr{L}^{2} .
$$

It can be seen from (3.25), (3.20) and (3.11) that $S(\mathscr{L})$ as given by (3.24) is indeed of the form (3.22), the real coefficients of $1, \gamma^{K}, G^{P Q}$ being given 
by

$$
\left.\begin{array}{l}
F(\mathscr{L}), \\
\frac{1}{2} \varepsilon_{K L M P Q} \mathscr{L}^{L M} \mathscr{L}^{P Q}, \\
2\left[\mathscr{L}_{P J} \mathscr{L}^{J}{ }_{Q}-(1+\operatorname{Tr} \mathscr{L}) \mathscr{L}_{P Q}\right],
\end{array}\right\}
$$

apart from a factor $[16 F(\mathscr{L})]^{-1 / 2}$.

\section{Dirac matrix description of Lorentz transformations}

Let $x^{\mu}(\mu=1,2,3,0)$ be a point of $E_{3,1}$ (ordinary spacetime), and let the metric tensor be $g^{\mu \nu}$

$$
g^{\mu \nu}=0, \quad \mu \neq \nu ; \quad g^{11}=g^{22}=g^{33}=-g^{00}=-1 .
$$

The Lorentz group consists of those real transformations

$$
x^{\mu} \rightarrow \hat{x}^{\mu}=L^{\mu}{ }_{\nu} x^{\nu}
$$

in $E_{3,1}$, which leave $g_{\mu \nu} x^{\mu} x^{\nu}$ invariant, i. e. whose coefficients satisfy

which implies

$$
L^{\mu v}=\left(L^{-1}\right)^{\nu \mu}
$$

$$
\operatorname{det} L= \pm 1, \quad\left(L^{00}\right) \equiv 1 .
$$

We have considered the Dirac matrix description of the real proper orthochronous de Sitter group $L_{4,1}$ whose elements leave the form $g_{J K} x^{J} x^{K}$ in $E_{4,1}$ invariant. By considering the elements

$$
\mathscr{L}=\left(\frac{L \mid 0}{0 \mid 1}\right)
$$

of $L_{4,1}$, i.e. those which leave $x^{5}$ invariant, we obtain a treatment of the real proper orthochronous Lorentz group $L_{3,1}$ since $\operatorname{det} \mathscr{L}=1, \mathscr{L}^{00} \equiv 1$ translate into $\operatorname{det} L=1, L^{00} \equiv 1$. Similarly by considering elements of $L_{4,1}$ of the form

$$
\mathscr{L}=\left(\frac{L \mid 0}{0 \mid-1}\right)
$$

i.e. those which reverse $x^{5}$, we get a treatment of real improper orthochronous Lorentz transformations.

As a preliminary, we attend to a matter which is of major significance in its own right, translation of the $E_{4,1}$ discussion of Dirac matrices into space-time notation. It is to be stressed that what is involved is mere translation. However, the results which are obtained in a very brief and systematic way afford an extremely complete description of the algebra of Dirac matrices as used throughout relativistic quantum mechanics and field theory. Of the many results listed below, some are indeed both new and useful, but those which are not new, have seldom been derived in a systematic way and usually have involved much more algebraic effort. Of course, the systematic nature of the present discussion 
stems entirely from (2.3) which yields, in space-time notation, the results

and

$$
\begin{gathered}
\gamma^{\mu} \gamma^{\nu} \gamma^{\varrho}=g^{\mu \nu} \gamma^{\varrho}-g^{\mu \varrho} \gamma^{\nu}+g^{\nu \varrho} \gamma^{\mu}- \\
-\varepsilon^{\mu \nu \varrho \sigma} \gamma_{\sigma} \gamma_{5},
\end{gathered}
$$

$$
\gamma^{\mu} \gamma^{\nu} \gamma^{5}=g^{\mu \nu} \gamma^{5}-\frac{1}{2} \varepsilon^{\mu \nu \sigma \tau} \gamma_{o} \gamma_{\tau}
$$

All further results stem from these. However, it is faster to obtain the following by simplification of the equations of section two. Using the usual notation ${ }^{4}$

$$
\begin{aligned}
\sigma^{\mu \nu} & =\frac{1}{2} i\left[\gamma^{\mu}, \gamma^{v}\right], \\
\sigma^{\mu 5} & =\frac{1}{2} i\left[\gamma^{\mu}, \gamma^{5}\right]=i \gamma^{\mu} \gamma^{5},
\end{aligned}
$$

we can draw up a list of further multiplication rules

$$
\begin{aligned}
\sigma^{\mu \nu} \gamma^{\varrho}= & -i\left(g^{\mu \varrho} \gamma^{\nu}-g^{\nu \varrho} \gamma^{\mu}\right)-\varepsilon^{\mu \nu \varrho \lambda} \sigma_{\lambda 5}, \\
{\left[\sigma^{\mu \nu}, \gamma^{\varrho}\right]=} & -2 i\left(g^{\mu \varrho} \gamma^{\nu}-g^{\varrho} \gamma^{\mu}\right) \\
\sigma^{\mu \nu} \gamma^{5}= & -\frac{1}{2} \varepsilon^{\mu \nu \varrho \lambda} \sigma_{\varrho \lambda} \\
{\left[\sigma^{\mu \nu}, \gamma^{5}\right]=} & 0, \\
\gamma^{\mu} \sigma^{\nu 5}= & i g^{\mu \nu} \gamma^{5}-\frac{1}{2} \varepsilon^{\mu \nu \varrho \lambda} \sigma_{\varrho \lambda}, \\
{\left[\gamma^{\mu}, \sigma^{\nu 5}\right]=} & 0, \\
\gamma^{\mu} \gamma^{\nu} \gamma^{\varrho} \gamma^{\lambda}= & g^{\mu \nu} \gamma^{\varrho} \gamma^{\lambda}-g^{\mu \varrho} \gamma^{\nu} \gamma^{\lambda}+g^{\mu \lambda} \gamma^{\nu} \gamma^{\varrho} \\
& +g^{\varrho \lambda} \gamma^{\mu} \gamma^{\nu}-g^{\nu \lambda} \gamma^{\mu} \gamma^{\varrho}+g^{\nu \varrho} \gamma^{\mu} \gamma^{\lambda} \\
& -g^{\mu \nu} g^{\varrho \lambda}+g^{\mu \varrho} g^{\nu \lambda}-g^{\mu \lambda} g^{\nu \varrho}-\varepsilon^{\mu \nu \varrho \lambda} \gamma^{5}, \\
\gamma^{\mu} \gamma^{\nu} \gamma^{\varrho} \gamma^{5}= & g^{\mu \nu} \gamma^{\varrho} \gamma^{5}-g^{\mu \varrho} \gamma^{\nu} \gamma^{5}+g^{\nu \varrho} \gamma^{\mu} \gamma^{5} \\
& -\varepsilon^{\mu \nu \varrho \lambda} \gamma_{\lambda}, \\
\sigma^{\mu \nu} \sigma^{\varrho \lambda}= & i\left(g^{\nu \varrho} \sigma^{\mu \lambda}+g^{\mu \lambda} \sigma^{\nu \varrho}-g^{\mu \varrho} \sigma^{\nu \lambda}-g^{\nu \lambda} \sigma^{\mu v}\right) \\
+ & g^{\mu \varrho} g^{\nu \lambda}-g^{\mu \lambda} g^{\nu \varrho}+\varepsilon^{\mu \nu \varrho \lambda} \gamma^{5}, \\
{\left[\sigma^{\mu \nu}, \sigma^{\varrho \lambda}\right]=} & 2 i\left(g^{\nu \varrho} \sigma^{\mu \lambda}+g^{\mu \lambda} \sigma^{\nu \varrho}-g^{\mu \varrho} \sigma^{\nu \lambda}-g^{\nu \lambda} \sigma^{\mu \varrho}\right) \\
\sigma^{\mu \nu} \sigma^{\varrho 5}= & -\sigma^{\varrho 5} \sigma^{\mu \nu} \\
= & i\left(g^{\nu \varrho} \sigma^{\mu 5}-g^{\mu \varrho} \sigma^{\nu 5}\right)+\varepsilon^{\mu \nu \varrho \lambda} \gamma_{\lambda}, \\
\sigma^{\mu 5} \sigma^{\nu 5}= & -\gamma^{\mu} \gamma^{\nu} .
\end{aligned}
$$

We may also list trace properties, best obtained usually by translation of those given in, or potentially available from section two. This

${ }^{4}$ We still have $\gamma_{5}=-\gamma^{5},\left(\gamma^{5}\right)^{2}=-1$, of course. 
gives a very rapid approach to results such as

$$
\begin{aligned}
& \operatorname{Tr}\left(\gamma^{\mu} \gamma^{\nu} \gamma^{\varrho} \gamma^{\sigma} \gamma^{5}\right)=4 \varepsilon^{\mu \nu \varrho \sigma} \\
& \operatorname{Tr}\left(\gamma^{\mu} \gamma^{\nu} \gamma^{\varrho} \gamma^{\sigma} \gamma^{\varkappa} \gamma^{\lambda} \gamma^{5}\right) \\
&= 4\left(g^{\mu \nu} \varepsilon^{\varrho \varkappa x \lambda}-g^{\mu \varrho} \varepsilon^{\nu \sigma \varkappa \lambda}+g^{\nu \varrho} \varepsilon^{\mu \sigma \varkappa \lambda}\right. \\
&+g^{\varrho \sigma} \varepsilon^{\mu \nu \varkappa \lambda}-g^{\nu \sigma} \varepsilon^{\mu \varrho \varkappa \lambda} \\
&\left.+g^{\mu \varrho} \varepsilon^{\nu \varkappa \lambda}+g^{\varkappa \lambda} \varepsilon^{\mu \nu \varrho \sigma}\right) .
\end{aligned}
$$

On the other hand possession of complete multiplication rules is very useful for direct trace calculation. For example, (4.13) allows one to turn knowledge of the fourfold trace into an explicit expression for an eight fold trace. However, we refrain from presenting this or more complicated formulae explicitly.

We now return to the specialization of the results of section three, that gives the Dirac matrix description of $L_{3,1}$. We have seen that the elements $\mathscr{L}$ of $L_{4,1}$ can be defined in terms of four by four matrices $S \in V S p_{4}$, which satisfy

together with

$$
S^{+} \gamma^{0} S=\gamma^{0},
$$

$$
\widetilde{S} B S=B,
$$

or equivalently

according to

$$
S^{*}=D S D^{-1}
$$

$$
\mathscr{L}(S)^{K} \gamma^{J}=S \gamma^{K} S^{-1} .
$$

To find what further properties are possessed by the elements $S$ of $V S p_{4}$ which describe proper Lorentz transformations, we consider (4.21) for $\mathscr{L}$ such that

$$
\begin{aligned}
\mathscr{L}(S)^{J K} & \equiv L(S)^{\mu v}(J \neq 5 \equiv \mu, K \neq 5 \neq v), \\
\mathscr{L}(S)^{J 5} & =\mathscr{L}(S)^{5}=0 \quad(J, K \neq 5), \\
\mathscr{L}(S)^{55} & =1
\end{aligned}
$$

This yields the definition

$$
L(S)^{\mu \nu}=\frac{1}{2} \operatorname{Tr}\left(\gamma^{\mu} S \gamma^{\nu} S^{-1}\right)
$$

for $L \in L_{3,1}$ in terms of $S \in V S p_{4}$, as well as the $K=5$ equation

so that

$$
\gamma^{5}=S \gamma^{5} S^{-1},
$$

$$
\left[S, \gamma^{5}\right]=0
$$

is the required further property of those $S$ which correspond to elements of $L_{3,1}$. Of course, the definition (4.22) is well known, as indeed are (3.1), (3.3) and (4.23) although their role which we shall discuss below is not 
always adequately stressed. However, the inverse of (4.22), the explicit expression for the $S$, which obeys (3.1), (3.3) and (4.23) and corresponds to the general element $L \in L_{3,1}$, is new in the present treatment. By insertion of (4.21) into (3.24), we derive this result in the form

$$
\begin{aligned}
S \equiv & S(L) \\
= & {\left[G(L)-\frac{1}{2} \varepsilon_{\mu \nu \varrho \sigma} L^{\mu \nu} L^{\varrho \sigma} \gamma^{5}+i \Gamma\left(L^{2}\right)\right.} \\
& -i(2+\operatorname{Tr} L) \Gamma(L)] /[16 G(L)]^{1 / 2},
\end{aligned}
$$

where

$$
\begin{gathered}
G(L)=2(1+\operatorname{Tr} L)+\frac{1}{2}(\operatorname{Tr} L)^{2}-\frac{1}{2} \operatorname{Tr} L^{2}, \\
\Gamma(L)=L_{\varkappa \lambda} \sigma^{\varkappa \lambda} .
\end{gathered}
$$

The restriction (4.23) on $S$ herein reflects itself in the absence of $\gamma^{\mu}$, $\sigma^{\mu 5}$ terms in (4.24). It can, of course, be established that (4.24) yields the well-known forms for $S(L)$ corresponding to $L$ a spatial rotation or a "pure" Lorentz transformation. In the case of a rotation $\theta$ in a positive sense about a unit spatial vector $\mathrm{n}$, one inserts $L^{00}=1, L^{0 k}=L^{k 0}=0$

$$
-L^{j k}=R^{j k}=\cos \theta \delta^{j k}+(1-\cos \theta) n^{j} n^{k}-\sin \theta \varepsilon^{j k \imath} n^{l}
$$

into (4.1), i.e. $x^{0} \rightarrow \hat{x}^{0}=x^{0}, x^{j} \rightarrow \hat{x}^{j}=R^{j k} x^{k}$.

Then using the identity [11]

$$
(\operatorname{Tr} R) 1-\widetilde{R}=(\operatorname{Tr} R) R-R^{2},
$$

we can simplify (4.24) into the forms

$$
\begin{gathered}
S(\mathbf{n}, \theta)=\frac{\left.[\mathbf{1}+\operatorname{Tr} R)-i \sigma^{i j} R^{i j}\right]}{[4(1+\operatorname{Tr} R)]^{1 / 2}}, \\
=\cos \frac{\theta}{2}-i \sum \cdot \mathbf{n} \sin \frac{\theta}{2}
\end{gathered}
$$

where $2 \Sigma^{k}=\varepsilon^{i j k} \sigma^{i j}$. In the case of a pure Lorentz transformation of velocity $v=\tan h \chi$ in the direction of a unit spatial vector $\mathbf{m}$, we use (4.1) with $L^{00}=\cosh \chi, \quad L^{k 0}=-L^{0 k}=m^{k} \sin h \chi, \quad$ and $L^{i j}=g^{i j}+$ $+m^{i} m^{j}(1-\cos h \chi)$, and get the result

with $\alpha^{k}=\gamma^{0} \gamma^{k}$

$$
S(\chi, \mathbf{m})=\cos h \frac{\chi}{2}+\alpha \cdot \mathbf{n} \sin h \frac{\chi}{2}
$$

If we return to where we obtained the Dirac matrix description of proper orthochronous Lorentz transformations by looking at those of elements $\mathscr{L}$ of $L_{4,1}$ of from (4.21), we can similarly discuss improper orthochronous Lorentz transformations by looking at elements $\mathscr{L}$ of $L_{4,1}$ of form

$$
\begin{aligned}
\mathscr{L}^{J K} & \equiv L^{\mu \nu}(J \neq 5 \equiv \mu, K \neq 5 \equiv v) \\
\mathscr{L}^{J 5} & =\mathscr{L}^{5 K}=0(J, K \neq 5) \\
\mathscr{L}^{55} & =-1
\end{aligned}
$$


This yields the definition (4.22) for improper orthochronous $L$ in terms of those $S \in V S p_{4}$ which also satisfy

$$
-\gamma^{5}=S \gamma^{5} S^{-1}
$$

It is almost unnecessary to point out that the work of this section is essentially equivalent to the standard textbook treatment of the matrix which enters, via

$$
L^{\mu}{ }_{\nu} \gamma^{\nu}=S^{-1} \gamma^{\mu} S
$$

the proof of the Lorentz invariance of the Dirac equation. Of course, there we meet (3.1) as a direct consequence of the definition (4.30) of $S$ for $L \in L_{3,1}$; (3.3) by requiring that Lorentz transformation and charge conjugation of the Dirac wave function should commute; and (4.23) as the consequence of (4.30) which tells us that $\bar{\psi} \gamma^{5} \psi$ is pseudoscalar. Here we have proved that four by four $S$ such that

$$
S^{-1}=\gamma^{0} S^{-1} \gamma^{0}, \quad S^{*}=D S D^{-1}, \quad S=\gamma^{5} 5\left(\gamma^{5}\right)^{-1},
$$

constitute a group homomorphic to $L_{3,1}$ realised explicitly by (4.24). It is of course a matter of opinion of to what extent it offers a major improvement on the usual discussion to view the Dirac matrix description of $L_{3,1}$ within the context of the classical homomorphism of $U S p_{4}$ and $R_{5}$. The role of the constraints on $S$ is probably clarified. The first one is a pseudo-unitarity condition implying $S \in V_{4}$, determined by our desire to work ultimately in pseudo-Euclidean spaces; the second is essentially a symplectic condition implying $S \in V S p_{4}$; and the third enters as we pass to the subgroup of $V S p_{4}$, which is homomorphic to $L_{3,1}$. Further, (4.24), a vital ingredient of a complete discussion, is to the best of the author's knowledge new.

\section{References}

[1] Pauli, W.: Ann. Inst. Henri Poincaré 6, 109 (1936).

[2] Good, M. H.: Rev. Mod. Phys. 27, 187 (1955).

[3] JAUCH, J. M., and F. RoHRLICH: Theroy of photons and electrons, Reading, Mass.: Addison Wesley 1955.

[4] Schweber, S. S.: Introduction to relativistic quantum field theory. Evanston, Ill.: Row, Peterson and Company 1961.

[5] Pursey, D. L.: Private communication (1958).

[6] Delbourgo, R., M. A. Rashid, A. Salam , and J. Strathdee: Trieste preprint (1965), Appendix.

[7] JACOBSON, N.: Lie algebras. New York: Interscience 1962.

[8] Behrends, R. E., J. Dreitlein, C. Fronsdal and B. W. Lee: Rev. Mod. Phys. 34, 1 (1962) Section VC.

[9] Pandit, L. K.: Nuovo Cimento Supp. 11, 147 (1959).

[10] Sudarshan, E. C. G.: Brandeis lectures in theoretical physics. 1961, Vol. 2. New York: Benjamin 1962.

[11] Macfarlane, A. J.: J. Math. Phys. 3, 116 (1962). 